\title{
A SYSTEM OPTIMIZATION MODEL OF ADOPTION OF A NEW INFRASTRUCTURE WITH MULTI-RESOURCE AND MULTI-DEMAND SITES
}

\author{
Yaru Zhang1, Huayi Chen ${ }^{1}$, Tieju Ma ${ }^{1,2, ~ * ~}$ \\ ${ }^{1}$ School of Business, East China University of Science and Technology \\ Meilong Road 130, Shanghai 200237, China \\ ${ }^{2}$ International Institute for Applied System Analysis \\ Schlosplatz 1 A-2361 Laxenburg, Austria
}

\begin{abstract}
This study develops a conceptual system optimization model of adoption of a new infrastructure technology with multiple resource sites and multiple demand sites. With the model, this paper analyzes how the adoption of a new infrastructure technology is influenced by heterogeneous distances between different resource-demand pairs, technological spillover among different resource-demand pairs, different demand dynamics, and different technological learning rates. The main findings of the study are: from the perspective of system optimization, (1) heterogeneous distances among different resource-demand pairs will result in different adoption time of a new infrastructure; (2) technological spillover among different resource-demand pairs will accelerate the adoption of a new infrastructure; (3) it is hard to say that higher demand will pull faster adoption of a new infrastructure, and the optimal time of adopting of a new infrastructure is very sensitive to its technological learning rate.
\end{abstract}

Keywords: System optimization model, technology adoption, new infrastructure

\section{Introduction}

Adoption of new technologies is recognized as an important driver of economic growth and competitive advantage (e.g., Kuan et. al 2015). Researchers have developed various technology adoption models, such as the technology adoption life cycle model (Rogers 1962), the Bass diffusion model (Bass 1969), the technology acceptance model (TAM) (Bagozzi et. al 1992, Davis 1989), and system optimization models of technology adoption (e.g, Messner \& Strubegger 1994, Seebregts 2001). Adoption of a new infrastructure technology, such as a UHV (ultra high voltage) transmission grid, commonly requires very high investment cost. The cost of establishing a new infrastructure could decrease in the future with technological learning effect as the experience of using the new technology accumulates (Arrow 1962, Arthur 1989). The cost reduction in the future relies on investment in the early stages of infrastructure development, and historical observations have shown that technological learning is quite uncertain (McDonald \& Schrattenholzer 2001). Adoption of a new infrastructure commonly accompanied with system reconfiguration. For example, adoption of a UHV grid is commonly associated with relocating coal power plants, i.e., coal power plants can be moved from demand sites to

\footnotetext{
${ }^{*}$ Corresponding author tjma@ecust.edu.cn; ma@iiasa.ac.at( $\bowtie)$

0086-21-64252015
} 
resource sites (e.g. Ma \& Chi, 2012, Zhuang \& Jiang 2009); adoption of a cloud computation infrastructure is associated with moving computation capacity from the end-use site to the cloud. In short, adoption of a new infrastructure is accompanied with uncertain technological learning in the time dimension and system reconfiguration in the spatial dimension. Thus it makes sense to analyze when and at what pace a new infrastructure should be implemented from a system optimization perspective.

Most system optimization models of technology adoption treat infrastructures as links among different technologies or activities, rarely as main objects under study. Ma and Chen (2015) developed a system optimization adoption model of a new infrastructure with uncertain technological learning and spatial reconfiguration. Their concept model assumes a new infrastructure can be adopted to replace an existing one. The new infrastructure has technological learning potential but it is uncertain, and with the adoption of the new infrastructure, a product producing technology can be relocated from the resource site to the demand site. There was only one resource site and one demand site in their model. In reality, it is most likely that there are multiple resource sites and multiple demand sites, and the distances between different resource sites to demand sites could be different. This paper extends the model with a single resource site and a single demand site into a model with multiple resource sites and multiple demand sites. The extension enables us to analyze how the following factors influence the adoption of a new infrastructure which was missing in the previous model.

\section{- Heterogeneous distances between} different resource-demand pairs. With different distance, the cost of establishing the new infrastructure between different resource-demand pairs could be different. Then what is the optimal time of adopting the new infrastructure between different resource-demand pairs from a system optimization perspective?
- Technological spillover among different resource-demand pairs. Different resource-demand pairs might adopt the new infrastructure technology at different pace. The experience in the new infrastructure technology accumulates in resource-demand pairs which adopt the new infrastructure earlier will benefit those pairs which adopt the new infrastructure later, i.e, the cost of establishing the new infrastructure will be lower in the pairs which adopt the new infrastructure later. Then how this technological spillover effect influences the adoption of the new infrastructure from a system optimization perspective?

In addition to exploring how the above two factors influence the adoption of a new infrastructure technology from a system optimization perspective, this paper also analyze how different demand dynamics, initial investment cost and technological learning rate of a new infrastructure technology influence the adoption of the new infrastructure with a optimization framework including multiple resource sites and multiple demand sites.

Diffusion of new technologies, especially a new infrastructure technology, commonly takes a long time (Grubler, 2004). The model presented in this paper is developed from a long-term perspective. The model and analysis presented in this paper do not aim to represent the reality in terms of technological or economic details; instead, it is mainly for heuristic purposes.

The rest of the paper is organized as follows. Section 2 introduces the optimization model with multiple resource sites and multiple demand sites. Section3 analyzes how the adoption of a new infrastructure is influenced by heterogeneous distances, technological spillover effect, demand dynamics, and so on. Section4 gives concluding remarks.

\section{The model}

\subsection{Model framework}

For the sake of transparency, the 
techno-economic system of our model is quite simple and stylized. The simplification also follows previous research on endogenous technological change models (e.g., Grubler and Gritevskyi 1998, Manne and Barreto 2002, Ma and Nakamori 2009, Chi et al. 2012). Fig. 1 gives an illustration of the model framework. In the model, the economy demands one kind of homogeneous good, for example, electricity. And the good can be generated with a producing technology from resources. There are multi-resource sites and multi-demand sites in the system. The left side of Fig. 1 lists resource sites, and the right side lists demand sites. T1 and T2 are the same technology but located in different places for producing the good from resources to satisfy the demand, for example, coal power plants which can generate electricity from coal resources to satisfy the demand for electricity. There are two types of infrastructure technologies. One is existing infrastructure which is denoted with T3 in Fig. 1, and the other is a new infrastructure technology which is denoted with T4 in Fig. 1. With the existing infrastructure (e.g, railways for transporting coal), the resource has to be transported to demand sites where it will be used as the input for T1. With the adoption of the new infrastructure (e.g, UHV transmission grid), the producing technology $\mathrm{T} 1$ can be moved from demand sites to the resource sites and thus becomes to T2 in Fig.1, and the good produced by $\mathrm{T} 2$ will be transported (or transmitted) to demand sites with the new infrastructure.

The model assume that the best match between different resource sites and demand sites is already known, that is to say, one demand site will provide the resource for one demand site, and one demand site will be served by one demand site. This assumption is for simplifying the model formulations and searching for optimal solutions. We will relax this assumption in our future work. We use $q_{k}(k=1, \cdots, n)$ to denote the distance between the resource site and demand site in the $k$ th resource-demand pair. Not losing generality, we assume $q_{1} \leq q_{2}<\ldots \leq q_{n}$.

The existing infrastructure $\mathrm{T} 3$ is mature without learning effect, while the new infrastructure T4 has learning potential which means its cost could decrease in the future, depend on accumulated adoption of it. The distances between different resource-demand pairs are different, and thus the efficiencies and costs of using the new infrastructure could be different. When conducting a system optimization, different resource-demand pairs will adopt the new infrastructure at different time with different pace. The technological learning gained in resource-demand pairs which adopted the new infrastructure earlier can be spillover to pairs adopting the new infrastructure later. That is to say, the cost of establishing the new infrastructure in resource-demand pairs which adopted the new infrastructure later will be lower than the initial cost of the new infrastructure.

Diffusion of a new technology commonly takes a long time. We assume the entire decision time horizon is composed of 10 decision intervals. A decision interval is the basic time unit for installing new capacities of technologies. We assume a decision interval as of 10 years, and thus the entire decision time horizon is of 100 years.

The model framework and main assumptions follow existing operational optimization models such as MESSAGE model (Messner \& Strubegger 1994) and the MARKAL model (Seebregts 2001). What is new in the model introduced in this paper is that we address the relocation of producing technologies with adoption of a new infrastructure with multiple resource sites and multiple demand sites 


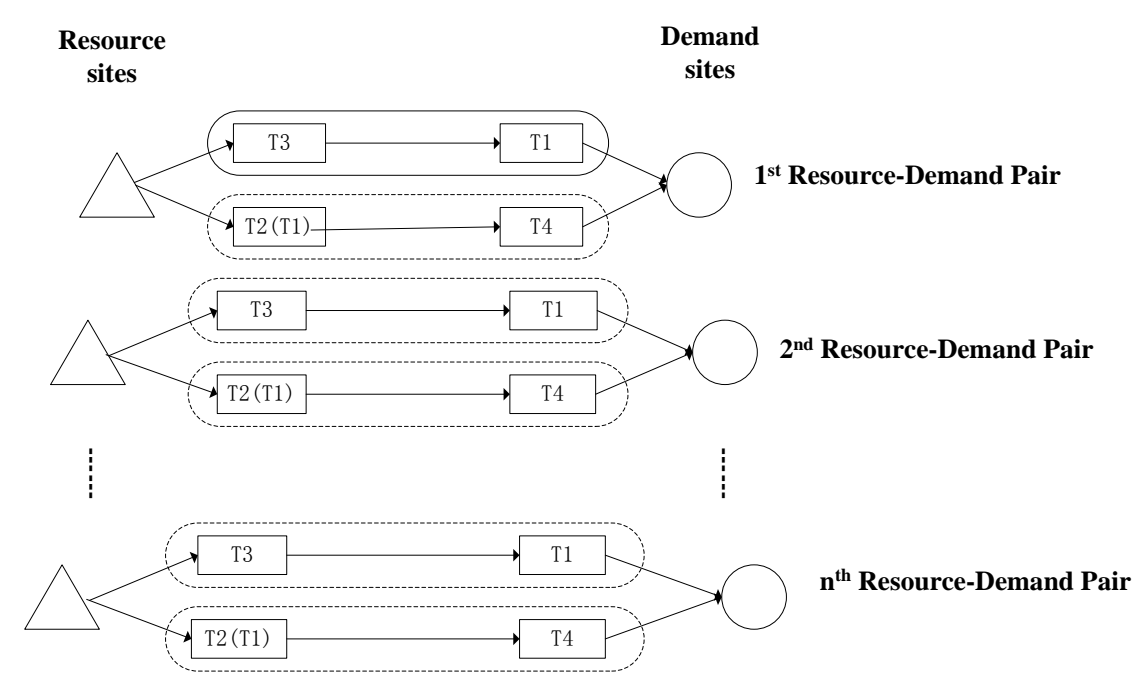

Note. T2/T1: Producing technology; T3: Existing infrastructure; T4: New infrastructure.

Figure 1 An illustration of the model framework

Table 1 Symbols for describing the model

\begin{tabular}{|c|c|}
\hline Symbols & Symbols meaning \\
\hline$q_{k}$ & Distance between the resource site and demand site in the $k$ th resource-demand pair \\
\hline$T 1(T 2)$ & Technology of producing the good from resource \\
\hline T3 & Existing infrastructure technology \\
\hline$T 4$ & New infrastructure technology \\
\hline$t$ & Time period $($ year $=t \times 10+2000, t=0,1, \cdots 10$ ) \\
\hline$d_{k}^{t}$ & Demand at time $\boldsymbol{t}$ in the $k$ th demand site \\
\hline$C_{E k}^{t}$ & Resource extraction at time step $t$ in the $k$ th resource site \\
\hline$r_{k}^{t}$ & Cumulative extraction of resource by time $t$ in the $k$ th resource site \\
\hline$\eta_{i k}$ & Efficiency of technology $i(i=1,2,3,4)$ in the $k$ th resource-demand pair \\
\hline$C_{i k}^{t}$ & Total installed capacity of technology $\boldsymbol{i}$ at time $\boldsymbol{t}$ in the $k$ th resource-demand pair \\
\hline$C_{i}^{t}$ & Total installed capacity of technology $\boldsymbol{i}$ at time $\boldsymbol{t}$ \\
\hline $\bar{C}_{i k}^{t}$ & Experience in technology $\boldsymbol{i}$ by time $\boldsymbol{t}$ in the $k$ th resource-demand pair \\
\hline$C_{\text {Fik }}^{t}$ & Unit investment cost of technology $\boldsymbol{i}$ at time $\boldsymbol{t}$ in the $k$ th resource-demand pair \\
\hline$b_{4}$ & $\begin{array}{l}\text { Elasticity of the unit investment cost of the new infrastructure with regard to its cumulative } \\
\text { installed capacity }\end{array}$ \\
\hline$C_{O M i}$ & Operation and maintenance cost of technology $\boldsymbol{i}$ \\
\hline$\tau_{i}$ & Plant life of technology $\boldsymbol{i}$ \\
\hline$\delta$ & Discount rate \\
\hline$\theta$ & Technological spillover rate \\
\hline \multicolumn{2}{|c|}{ Decision Variables: } \\
\hline$x_{i k}^{t}$ & Output of technology $\boldsymbol{i}$ at time $\mathrm{t}$ in the kth resource-demand pair \\
\hline$y_{i k}^{t}$ & New installation of technology $\boldsymbol{i}$ at time $\boldsymbol{t}$ in the kth resource-demand pair \\
\hline
\end{tabular}




\subsection{Model formulations}

Table 1 introduces the meaning of symbols which will be used to describe the model.
The objective of the model is to minimize the total cost of the system while satisfying dynamic demand from a long term perspective. Eq. (1) is the objective function of the model.

$$
\min \sum_{k=1}^{n} \sum_{t=1}^{T} \sum_{i=1}^{4}\left(\frac{1}{1+\delta}\right)^{t}\left(c_{F i k}^{t} y_{i k}^{t}+c_{O M i} x_{i k}^{t}\right)+\sum_{k=1}^{n} \sum_{t=1}^{T}\left(\frac{1}{1+\delta}\right)^{t} c_{E}^{t} r_{k}^{t}
$$

The total cost of the system includes two items. The first item of the objective function includes the investment cost of building new capacities and the O\&M (operation and maintenance) cost. The second term represents the cost of extracting resource.

With technological learning, the unit investment cost $c_{F 4 k}^{t}$ of the new infrastructure in Eq. (1) will decrease as a function of cumulative installed capacity with $b_{4}$, as shown in Eq.(2).

$$
c_{F 4 k}^{t}=c_{F 4 k}^{0} \times\left(\bar{C}_{4 k}^{t}\right)^{-b_{4}}
$$

There is technological spillover effect. The technological learning gained in resource-demand pairs which adopted the new infrastructure earlier can be spilled over to pairs adopting the new infrastructure later. The $\bar{C}_{4 k}^{t}$ in Eq. (2) is the sum of experience (quantified with cumulative installed capacity) gained in the $k$ th resource-demand pair and experience spilled over from other resource-demand pairs, as shown in Eq. (3)

$$
\bar{C}_{4 k}^{t}=\sum_{j=1}^{t} C_{4 k}^{j}+\theta \hat{C}^{t}
$$

where $\hat{C}^{t}$ is the experience spilled over from other resource-demand pairs. The $C_{4 k}^{j}$ in Eq. (3) is a function of previous decision on adopting the new infrastructure, and

$$
C_{4 k}^{j}=\sum_{h=j-\tau_{i}}^{j} y_{4 k}^{h}
$$

T1/T2 and T3 are assumed mature without learning potential, and so $c_{F i k}^{t}(i=1,2,3)$ in Eq. (1) are constant.
The extraction cost of resource increases over time as a function of resource depletion, as shown in Eq. (5).

$$
c_{E k}^{t}=c_{E k}^{0}+\beta{\overline{r_{k}}}^{t},
$$

where $\beta$ is a constant coefficient, and

$$
\begin{gathered}
\bar{r}_{k}{ }^{t}=\sum_{j=1}^{t} r_{k}^{j}, \\
r_{k}^{j}=\frac{x_{1 k}^{j}}{\eta_{1 k} \times \eta_{3 k}}+\frac{x_{2 k}^{j}}{\eta_{2 k}},
\end{gathered}
$$

where $\eta_{1 k}=\eta_{2 k}$ because $\mathrm{T} 1$ and $\mathrm{T} 2$ are the same technology located at different places.

The objective function is subject to several sets of constraints. The first set represents demand constraints. Eq. (8) denotes that the demand at each demand site must be satisfied at each decision interval.

$$
x_{1 k}^{t}+\eta_{4 k} x_{2 k}^{t} \geq d_{k}^{t}
$$

The second set includes balance constraints. Eq. (9) indicates that the maximum input of T1 is less than or equal to the output of T3. Eq. (10) denotes that the maximum input of $\mathrm{T} 4$ is less than or equal to the output of $\mathrm{T} 2$.

$$
\begin{gathered}
x_{3 k}^{t} \eta_{1 k} \geq x_{1 k}^{t} \\
x_{2 k}^{t} \eta_{4 k} \geq x_{4 k}^{t}
\end{gathered}
$$

The third set represents capacity constraints. Eq.(11) denotes that the production of each technology cannot go beyond its total installed capacity at each decision interval.

$$
C_{i k}^{t} \geq x_{i k}^{t}
$$


The fourth set constraints contain the decision variables. Non-negativity constraints are placed on the decision variables, as shown in Eq. (12) and (13).

$$
\begin{aligned}
& x_{i k}^{t} \geq 0 \\
& y_{i k}^{t} \geq 0
\end{aligned}
$$

The efficiency of the new infrastructure will decrease as the distance increases. We analyze the adoption of the new infrastructure with three types of dynamics of $\eta_{4 k}$ which is a function of the distance between a resource site and a demand site, as shown in Fig. 2. In all of the three types of efficiency dynamics, the value of efficiency is from 0.7 to 1 . The three types efficiency dynamics are namely E1, E2, and E3, which are described with Eq.(14), Eq.(15), and Eq.(16), respectively. As shown in Fig.2, T4's efficiency with E2 is always higher than that with E1 and E3.

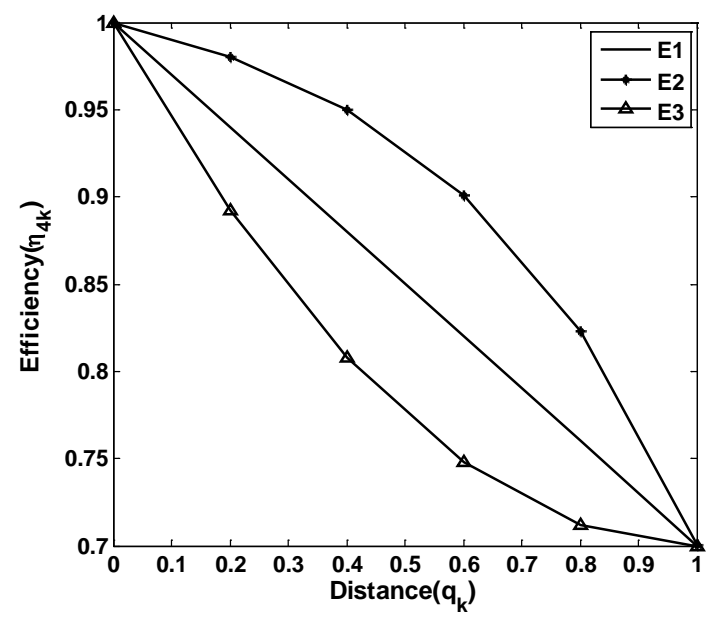

Figure 2 Three types of efficiency dynamics of the new infrastructure

$$
\text { E1: } \eta_{4 k}=1-0.3 \times q_{k}
$$

E2: $\eta_{4 k}=-0.0333 \times 10^{q_{k}}+1.0333$

$$
\text { E3: } \eta_{4 k}=0.3 \times q_{k}^{2}-0.6 \times q_{k}+1
$$

The demand in the model is exogenous and change over time. We analyze the adoption of the new infrastructure under three scenarios of demand dynamics, namely D1, D2, and D3, which are described with Eq. (17), Eq. (18), and Eq. (19), respectively. As shown in Fig. 3, with $\mathrm{D} 1$, the demand at each demand site grows very slowly at the beginning, and then it grows faster; with D2, the demand grows slowly at the beginning, then it grows faster, and then it grows slowly again; with D3, the demand grows very fast at the beginning, then the growth rate starts to decrease, and finally the demand starts to decrease.

$$
\begin{gathered}
\text { D1: } \quad d_{k}^{t}=d_{k}^{0} \times(1+0.05)^{t} \\
\text { D2: } \quad d_{k}^{t}=\frac{106 \times d_{k}^{0}}{1+106 \times e^{-0.08 \times t}} \\
\text { D3: } \quad d_{k}^{t}=d_{k}^{0}-1060 \times t^{2}+142000 \times t
\end{gathered}
$$

In the following, we present optimization results of the model in different scenarios and explore how the distance, spillover effect, demand, initial investment cost, and learning rate influence the adoption of the new infrastructure technology.

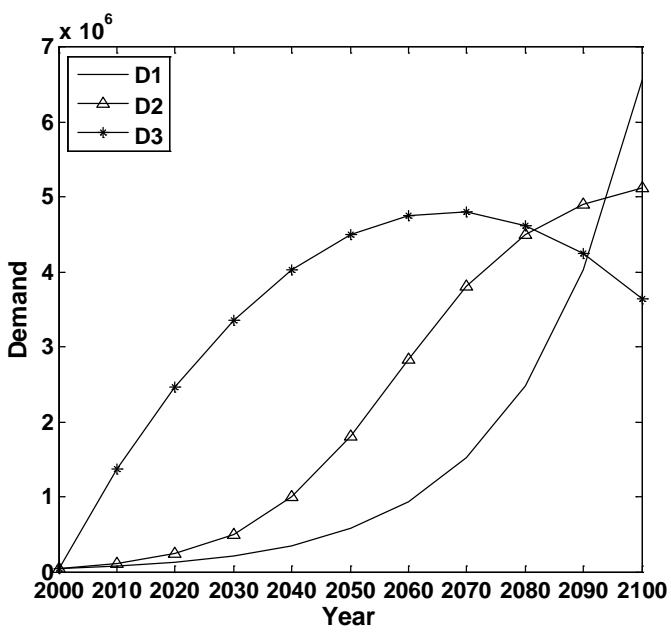

Figure 3 Three types of demand dynamics

\section{Simulations and analysis}

\subsection{Adoption of the new infrastructure with a baseline simulation}

We assume there are 5 resource sites and 5 demand sites. One resource site corresponds to 
one demand site, which means there are 5 resource-demand pairs in the entire system. The value of distance between resource-demand pairs are $q_{1}=0.2, q_{2}=0.4, q_{3}=0.6, q_{4}=0.8$, $q_{5}=1$, respectively. For exploring how the adoption of the new infrastructure technology is influenced by different factors, we first do a base line simulation with the model and then conduct simulations with different parameter values. Parameter values as well as efficiency and demand dynamics in the baseline simulation are presented in Table 2.

Table 2 Parameter values in the baseline simulation

\begin{tabular}{lllll}
\hline Parameters & $\mathrm{T} 1$ & $\mathrm{~T} 2$ & $\mathrm{~T} 3$ & $\mathrm{~T} 4$ \\
\hline Initial investment cost (US\$/kilowatt) $\left(c_{F i k}^{0}\right)$ & 500 & 500 & 100 & 30000 \\
Efficiency $\left(\eta_{i k}\right)$ & 0.4 & 0.4 & 0.85 & 0.9 \\
Plant life $($ year $)\left(\tau_{i}\right)$ & 30 & 30 & 30 & 30 \\
Initial total installed capacity (kilowatt) $\left(C_{i k}^{0}\right)$ & 50000 & 0 & 0 & 0 \\
O+M cost (US\$/kilowatt Year) $\left(C_{\text {OMi }}\right)$ & 200 & 200 & 80 & 100 \\
learning rate $\left(1-2^{-b_{i}}\right)$ & 0 & 0 & 0 & 0.2 \\
\hline Initial demand $d_{k}^{0}$ & 50000 & & & \\
Initial extracting cost(US\$/kilowatt) $\left(c_{E k}^{0}\right)$ & 15 & & & \\
Extraction coefficient $(\beta)$ & $10^{-10}$ & & & \\
Discount rate $(\delta)$ & $5 \%$ & & \\
Spillover rate of technological learning $(\theta)$ & 1 & & \\
Efficiency dynamics & E1: Eq. (14) & \\
Demand dynamics & D1: Eq.(17) & \\
\hline
\end{tabular}

Fig. 4 shows the adoption of the new infrastructure technology in the five resource-demand pairs as well as the entire system with the baseline simulation, from which we can see that the longer the distance is, the later the adoption is. The new infrastructure technology dominates the $1^{\text {st }}$ resource-demand pair from 2050, the $2^{\text {nd }}$ pair from 2060, the $3^{\text {rd }}$ and the $4^{\text {th }}$ pairs from 2070, and it does not appear in the $5^{\text {th }}$ pair at all which is of the longest distance. This is because with the longer distance, the lower the efficiency of the new infrastructure is, and thus it becomes more and more uneconomic to adopt the new infrastructure with the increase of distance. The resource-demand pair with the shortest distance adopts the new infrastructure firstly, with technological learning, the cost of adopting the new infrastructure decreases, and then it becomes economic for resource-demand pairs with longer distance and they start to adopt the new infrastructure.

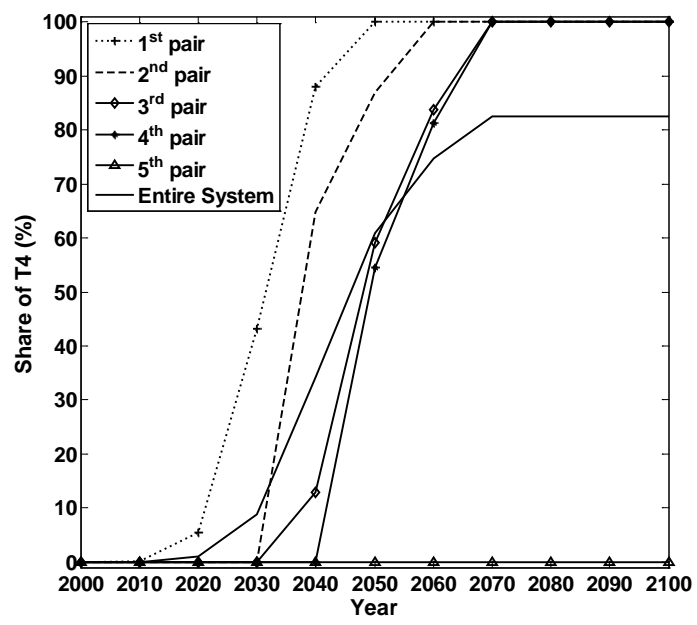

Figure 4 Adoption of T4 with the baseline simulation 


\subsection{Adoption of the new infrastructure with different technological spillover rate}

In the baseline simulation, the technological spillover rate $(\theta)$ is assumed to be 1 , which means the experience gained in one resource-demand pair can be spilled over to others completely. In order to explore how different spillover effect influence the adoption of the new infrastructure, we conducted simulations with different spillover rate values, $\theta=0.8$ and $\theta=0.5$. The left part of Fig. 5 plots the adoption of the new infrastructure in the five resource-demand pairs with $\theta=0.8$, and the right part of Fig. 5 plots that with $\theta=0.5$. From Fig. 5 we can see that the lower the spillover rate is, the later the adoption of the new infrastructure is. With $\theta=0.8$, the adoption of the new infrastructure in the first three resource-demand pairs is similar with that with $\theta=1$, and it is 10 years late in the $4^{\text {th }}$ resource-demand pair; with $\theta=0.5$, the adoption of the new infrastructure in the first four resource-demand pairs is $20-30$ years late than that with $\theta=1$. The new infrastructure does not appear in the 5th resource-demand pairs with either $\theta=0.8$ or $\theta=0.5$.
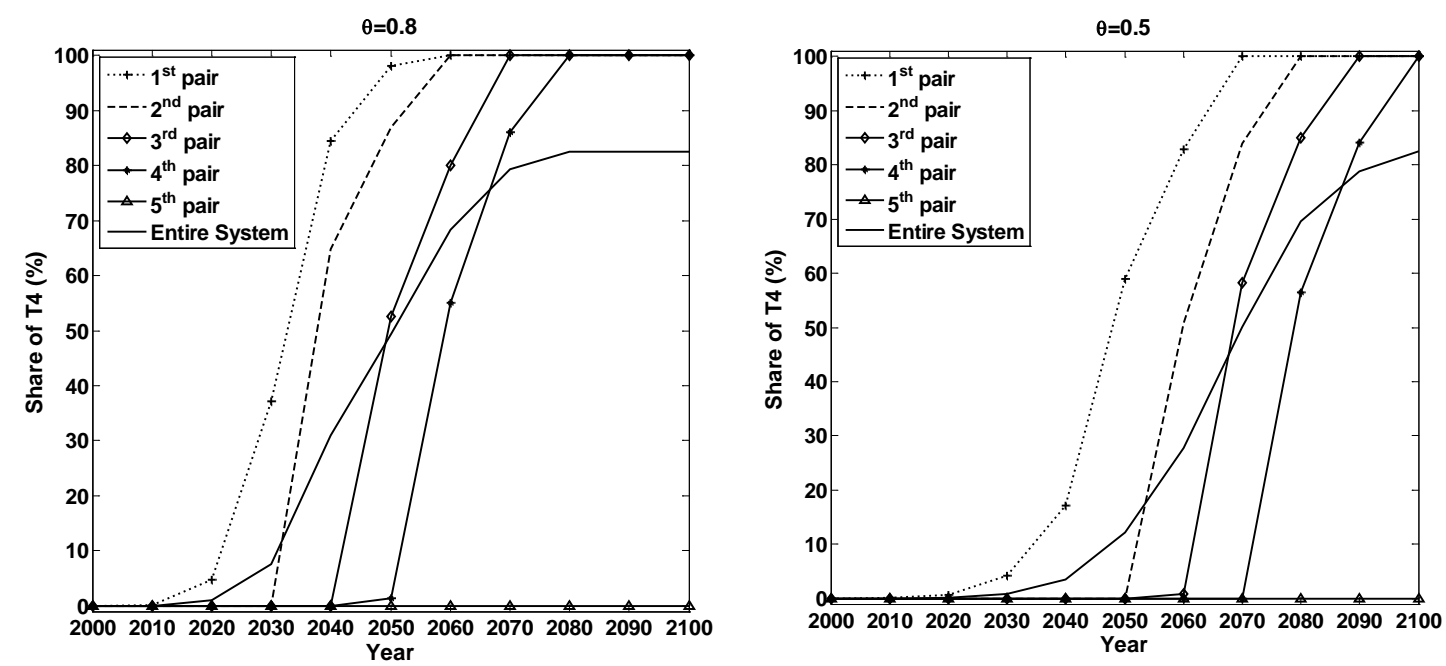

Figure 5 Adoption of T4 with different technological spillover rate

\subsection{The adoption of the new infrastructure with different efficiency dynamics}

In the baseline simulation, the efficiency of the new infrastructure decrease with E1 -- a linear function of its implemented distance, as shown in Eq. (14). For exploring how different efficiency dynamics influence the adoption of the new infrastructure, we conducted simulations with efficiency dynamics E2 -- an exponential function of the distance, and E3 -- a quadratic function of the distance. E2 and E3 are described with Eq. (15) and Eq. (16), respectively.

The left part of Fig. 6 plots the adoption of the new infrastructure in the five resource-demand pairs with $\mathrm{E} 2$, and the right part plot that with E3. As we can see, with E2, the new infrastructure does not appear in the $5^{\text {th }}$ resource-demand pair, the same as that with $\mathrm{E} 1$, but the adoption of the new infrastructure in the $2^{\text {nd }}$, the $3^{\text {rd }}$, and the $4^{\text {th }}$ resource-demand pairs are brought forward for around 10 years; with E3, the new infrastructure does not appear in the $3^{\text {rd }}$, $4^{\text {th }}$, and $5^{\text {th }}$ resource-demand pairs, and the adoption of the new infrastructure in the $1^{\text {st }}$ and $2^{\text {nd }}$ resource-demand pairs are around 20-40 years late than that with E1. In a summary, for the earlier adoption of the new infrastructure, $\mathrm{E} 2 \succ \mathrm{E} 1 \succ \mathrm{E} 3$, this is because with $\mathrm{E} 2$, the 
efficiency is always higher than that with E1 and E3 for any given distance, and with E1, it is higher than that with E3, as shown in Fig. 2. With any of the efficiency dynamics, the new infrastructure intends to be adopted with resource-demand pairs with short distances firstly, and then those with the longer distances. This is because with any of the efficiency dynamics, the longer the distance, the lower the new infrastructure's efficiency is and thus the more uneconomic it is.
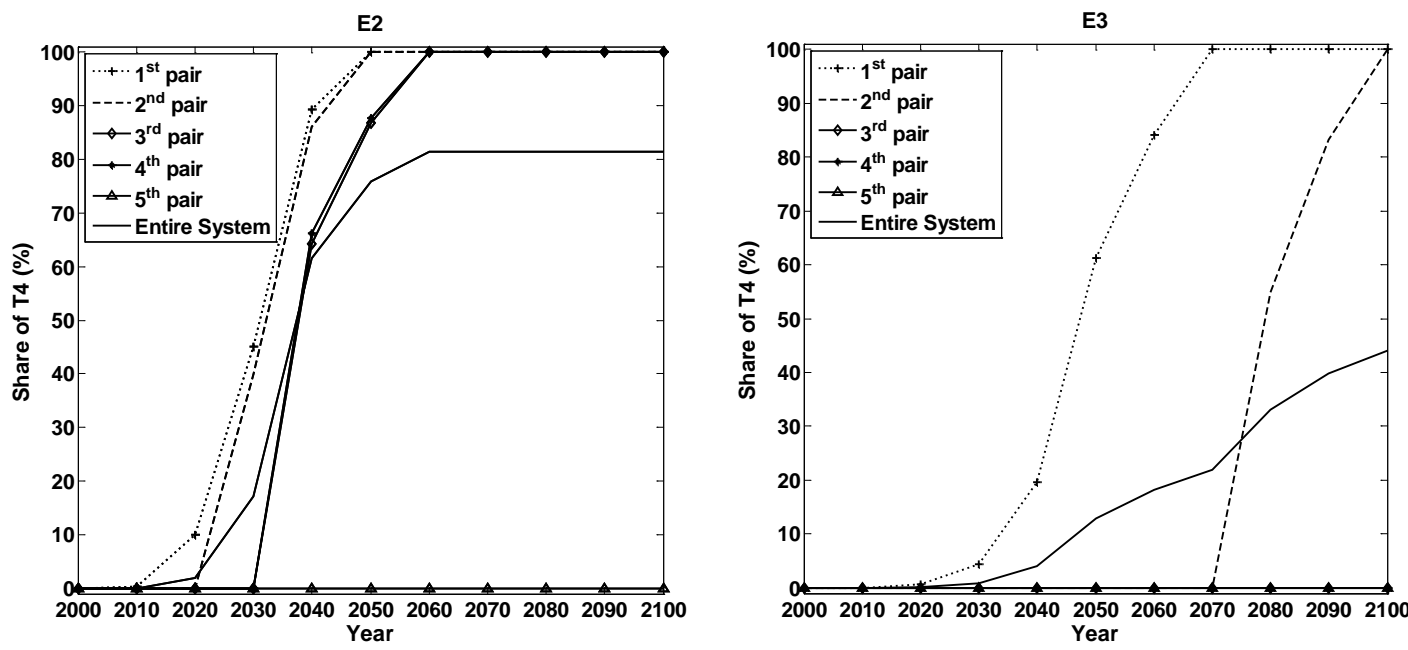

Figure 6 Adoption of T4 with different efficiency dynamics

\subsection{The adoption of the new infrastructure with different demand dynamics}

In the baseline simulation, the demand at each demand site increases with a constant annual growth rate (5\%), as shown in Eq. (16). For exploring how different demand dynamics influence the adoption of the new infrastructure, we conducted simulations with demand dynamics D2 -- a logistic function of time, and D3 -- a quadratic function of time. D2 and D3 are described with Eq. (17) and Eq. (18), respectively.

The left part of Fig.7 plots adoption of the new infrastructure in the five resource-demand sites with demand dynamics D2, and the right part of Fig. 7 plot that with demand dynamics D3. As we can see from Fig. 7, with demand dynamics D2, the new infrastructure will not be adopted at all; and with demand dynamics D3, the adoption of the new infrastructure is similar to that in the baseline simulation, i.e., with demand dynamics D1. From Fig. 3, we can see that for any given time before 2070, D3>D2>D1, but this does not result in that higher demand pulls earlier adoption of the new infrastructure, with other parameter values and dynamics as the same. Although D2 is higher than D1 before 2070, the new infrastructure is not adopted with D2. Although D1 is much higher than D3 after 2090, it does not result in much earlier adoption of the new infrastructure. With the three demand dynamics, we can hardly conclude what kind of demand dynamics will induce the fastest adoption of the new infrastructure. What we can conclude is that higher demand does not have to pull earlier adoption of the new infrastructure, at least for a certain time period. In our future work, we will explore in details how different demand dynamics influence the adoption of a new technology. 

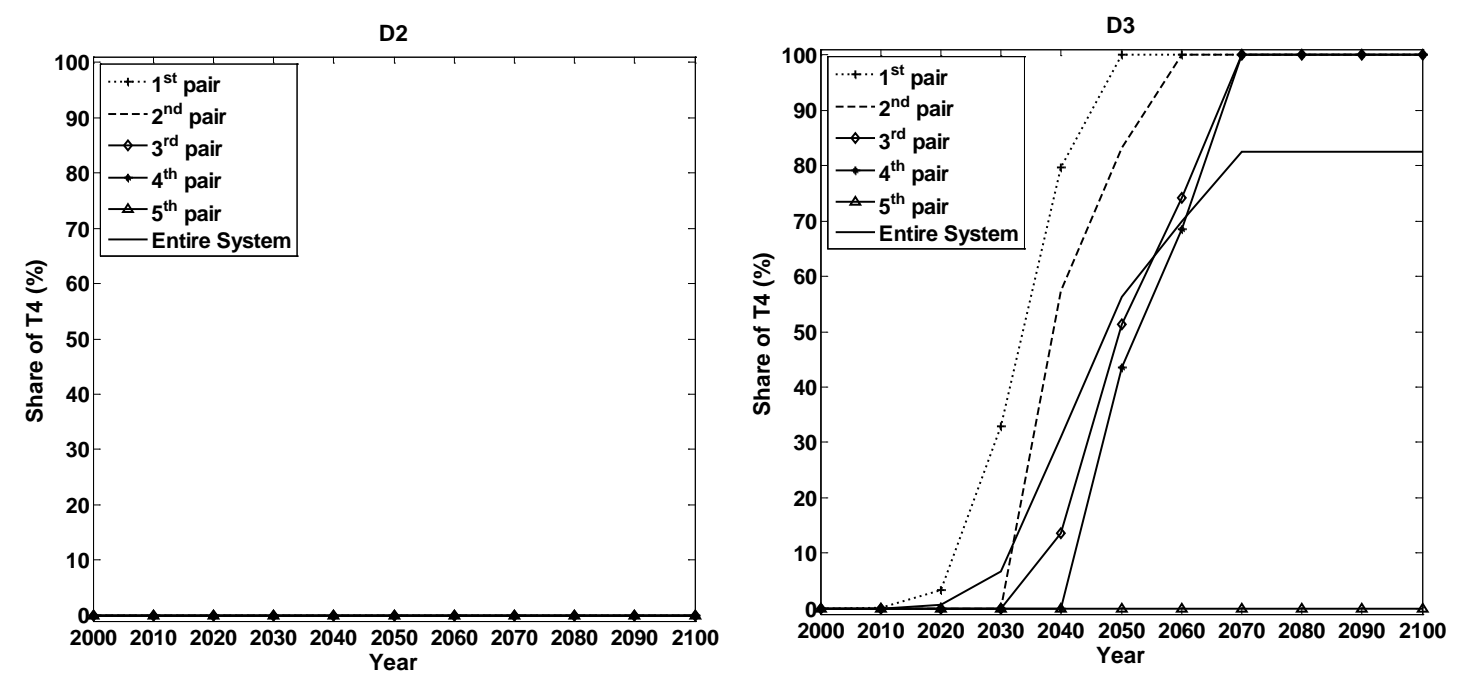

Figure 7 Adoption of $\mathrm{T4}$ with different demand dynamics

\subsection{The adoption of the new infrastructure with different investment cost}

In the baseline simulation, the investment cost of building the new infrastructure is assumed not related to its implemented distance. In this subsection, we assume the investment cost of the new infrastructure is a linear function of the distance with Eq. (20).

$$
c_{F i k}^{t}=c_{F i 1}^{t} \frac{q_{k}}{q_{1}},
$$

With Eq. (20), the longer the distance is, the higher the investment cost is. In the baseline simulation, the new infrastructure's efficiency will decrease with the increase of the distance. For exploring how dynamic investment costs as a function distance influence the adoption of the new infrastructure, we run a simulation with the new infrastructure's efficiency as a constant value (0.9), i.e., not influenced by its implemented distance. The left part of Fig. 8 plots the adoption of the new infrastructure in the five resource-demand pairs in this simulation. As we can see, in this simulation, the longer the distance is, the later the adoption of the new infrastructure is. This is because, the longer the distance is, the more uneconomic is to adopt the new infrastructure early. It is after experience in the new infrastructure is accumulated in the resource-demand pairs with short distance to reduce the investment cost with technological learning effect, then it becomes economic to adopt the new infrastructure in the resource-demand pairs with longer distances.

We also run a simulation with both the new infrastructure's investment cost and its efficiency as functions of its implemented distance, i.e., with both Eq. (20) and Eq. (14). The right part of Fig. 8 plots the adoption of the new infrastructure in the five resource-demand pairs in this simulation, from which we can see that the adoption in the $2^{\text {nd }}$ and the $3^{\text {rd }}$ resource-demand pairs is postponed much and the new infrastructure does not appear in the $4^{\text {th }}$ and the $5^{\text {th }}$ pairs. In this simulation, the influence of distance on the adoption of the new infrastructure is strengthened through both investment costs and efficiency. 

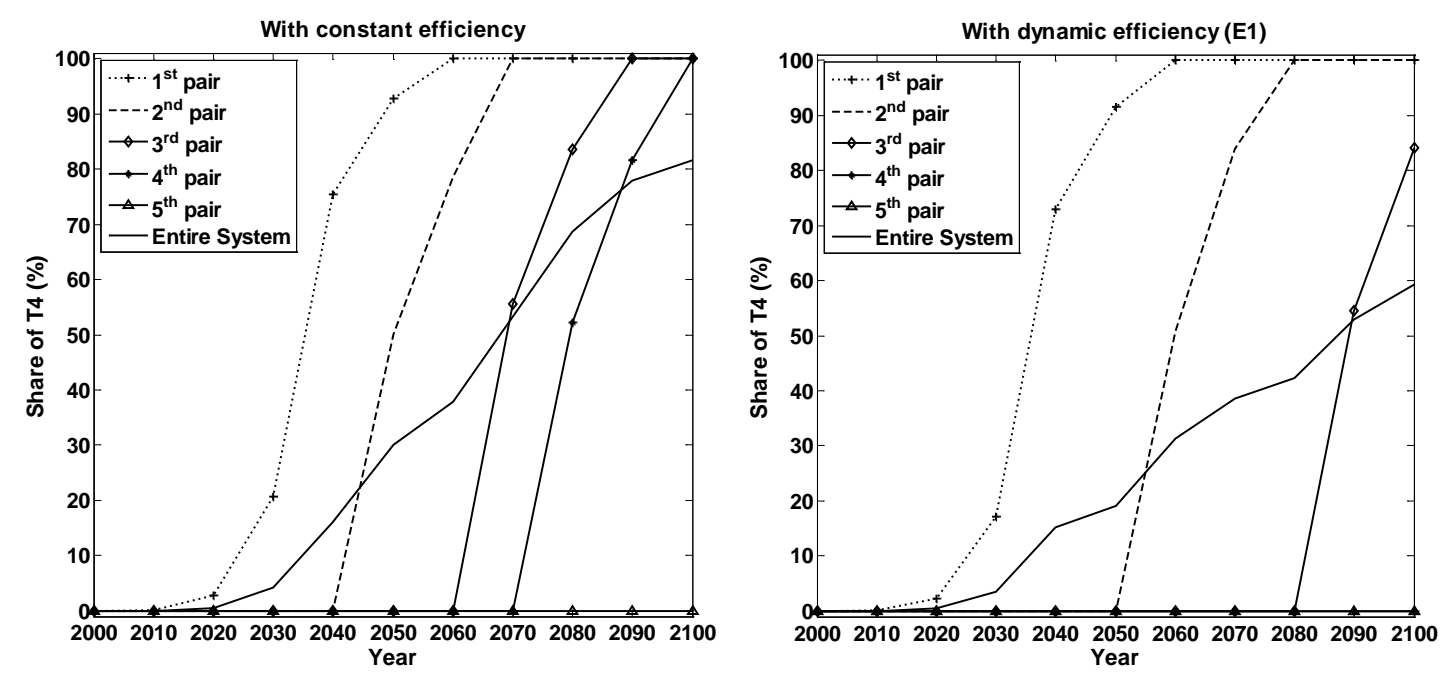

Figure 8 Adoption of T4 with different investment cost

\subsection{The adoption of the new infrastructure with different learning rate}

Technological learning is thought as the endogenous driving force for the adoption of currently uneconomic new technology. We run simulations with different technological learning rate of the new infrastructure to analyze how it influences the adoption of the new infrastructure. In the baseline simulation, the technological learning rate of the new infrastructure is assumed to be 20\%. The left part of Fig. 9 plots the adoption of the new infrastructure in the five resource-demand pairs with the technological learning rate as $18 \%$, with other parameter values and dynamics the same as in the baseline simulation, from which we can see that, the adoption of the new infrastructure in the $1^{\text {st }}$ to the $4^{\text {th }}$ resource-demand pairs is postponed for around 20 50 years. And the new infrastructure does not appear in the $5^{\text {th }}$ resource-demand pair.
The right part of Fig. 9 plots the adoption of the new infrastructure in the five resource-demand pairs with the technological learning rate as $22 \%$, with other parameter values and dynamics the same as in the baseline simulation, from which we can see that the adoption of the new infrastructure in the $2^{\text {nd }}$ to the $4^{\text {th }}$ resource-demand pairs is brought forward for about 10 20 years, and the $5^{\text {th }}$ resource-demand pair also adopts the new infrastructure which was not in all previous simulations presented in this paper. What we can summarize from the two simulations presented in Fig. 9 is that the model is very sensitive to the learning rate of the new infrastructure. Historical observations have shown that technological learning rates could be very uncertain (McDonald \& Schrattenholzer, 2001). In our future work, we plan to develop the model in to a stochastic optimization model to analyze what are the robust strategies of adopting the new infrastructure with uncertain technological learning. 

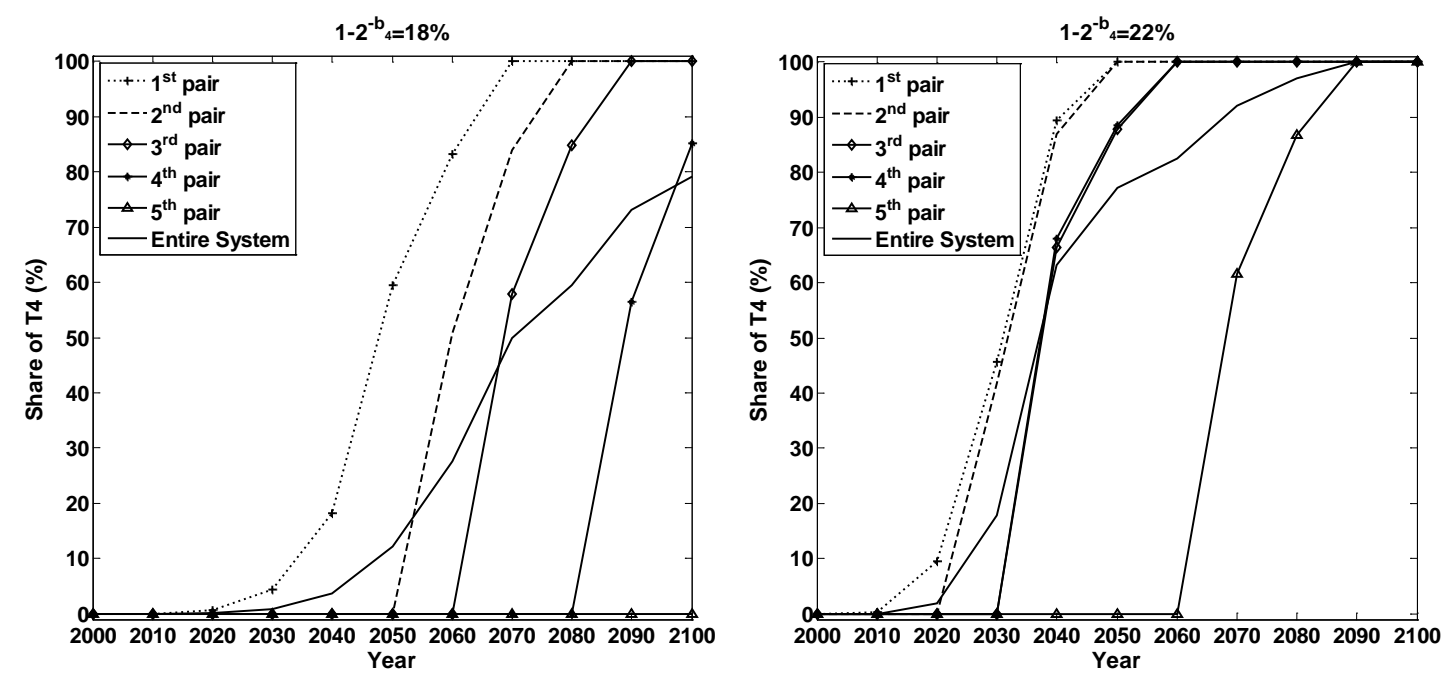

Figure 9 Adoption of T4 with different learning rate

\section{Conclusions}

This paper developed a conceptual system optimization model with multiple resource sites and multiple demand sites to study adoption of a new infrastructure technology. The main findings of the simulations presented in this paper include the following points:

(1) Heterogeneous distance among different resource-demand pairs will result in different adoption time of a new infrastructure because its investment cost might increase and its efficiency might decrease with its implemented distance increases. It is after experience in the new infrastructure is accumulated in the resource-demand pairs with short distance to reduce the investment cost with technological learning effect, then it becomes economic to adopt the new infrastructure in the resource-demand pairs with longer distances.

(2) Technological spillover among different resource-demand pairs will accelerate the adoption a new infrastructure, the higher the spillover effect, the faster the adoption is.

(3). From a system optimization perspective, it is hard to say that higher demand will pull faster adoption of a new infrastructure with our conceptual model, and the optimal time of adopting of a new infrastructure is very sensitive to its technological learning rate.

The policy implications of the above point (1) and point (2) are that authorities in planning the adoption of a new infrastructure should pay attention to heterogeneous distances among different resource-demand pairs, it is better to start with the most economic one to accumulate enough knowledge/experience, and it is important to promote the technological spillover among different resource-demand pairs.

The above point (3) implies that in our future work, it is necessary to do a more detailed analysis on demand dynamics and their influence on the adoption of a new infrastructure, and it makes sense to develop a stochastic optimization model to analyze the robust strategies on adoption a new infrastructure with uncertain technological learning.

\section{References}

[1] Kuan J, Rombe-Shulman S, Shittu E (2015) The political economy of technology adoption: The case of Saharan salt mining $[\mathrm{J}]$. The Extractive Industries and Society, 2015, 2(2): 328-338.

[2] Rogers, E. M. (1962) Diffusion of innovations. Glencoe: FreePress.

[3] Bass, F. (1969) A new product growth model for consumer durables. Management Science, 15(5), 215-227 [Management Science,50 (Number 12 Supplement), Dec 2004 ISSN 0025-1909, pp. 1833-1840].

[4] Bagozzi, R. P., Davis, F. D., Warshaw, P. R. 
(1992) Development and test of a theory of technological learning and usage. Human Relations, 45(7), 660-686.

[5] Davis, F.D. (1989) Perceived usefulness, perceived ease of use, and user acceptance of information technology. MIS Quarterly, 13(3), 319-340.

[6] Messner, S., \& Strubegger, M. (1994) The energy model MESSAGE III. In J. F. Hake, M. Kleemann, W. Kuckshinrichs, D. Martinsen, \& M. Walbeck (Eds.), Advances in systems analysis: Modelling energy-related emissions on a national and global scale (pp. 29-49). Jülich, Germany: Forschungszentrum Jülich.

[7] Seebregts, A. D. Energy/environmental modelling using the MARKAL family of models. In Proc. OR2001 conference, energy, and environment session, Duisburg, Germany, September 3-5.

[8] Arrow,K.J. (1962). The economic implications of learning by doing. Review of Economic Studies, 29(3), 155-173.

[9] Arthur,W.B.(1989) Competing technologies, increasing returns, and lock-in by historical events. Economic Journal, 99(394), 116-131.

[10] McDonald, A., \& Schrattenholzer, L. (2001). Learning rates for energy technologies. Energy Policy, 29(4), 255 261.

[11] Ma,T.J., Chi,C.J.(2012) Spatial configuration and technology strategy of China's green coal-electricity system. Journal of Renewable and Sustainable Energy,4,031806.doi: 10.10631/1.4730419.

[12] Zhuang X., jiang K.J (2009) Energy Content Analyses of Coal Product from Coal-mine to Consumer. Research and Approach, V01. 31(9 ):30-35.

[13] Ma T. J., Chen H. Y. (2015) Adoption of an emerging infrastructure with uncertain technological learning and spatial reconfiguration. European Journal of Operational Research, 243(3), 995-1003.
[14] Grubler, A. (2004) Technology and global change. Cambridge, UK: Cambridge University Press.

[15] Grubler, A., \& Gritsevskyi, A. (1998). A model of endogenous technological change through uncertain returns and learning (R\&D and investments). Working paper. IIASA, Laxenburg, Austria. $<$ http://www.iiasa.ac.at/Research/TNT/WE B/endog. pdf $>$.

[16] Manne, A. S., \& Barreto, L. (2002) Learning-by-doing and carbon dioxide abatement. Working paper. Stanford University, Stanford, CA.

[17] Ma,T.J., Grubler, A., Nakamori,Y. (2009) Modeling technology adoptions for sustainable development under increasing returns, uncertainty, and heterogeneous agents. European Journal of Operational Research, 195(1), 296-306. 